\title{
Monetäre Anreize zur Steuerung der Ladelast von Elektrofahrzeugen - eine modellgestützte Optimierung
}

\author{
Alexandra-Gwyn Paetz • Thomas Kaschub • \\ Martin Kopp • Patrick Jochem • Wolf Fichtner
}

Zusammenfassung Elektromobilität soll die Senkung der $\mathrm{CO}_{2}$-Emissionen im Transportsektor ermöglichen und damit einen Beitrag zu den klimapolitischen Zielen leisten. Eine verstärkte Verbreitung von Elektrofahrzeugen kann wesentliche Rückwirkungen auf das Elektrizitätssystem, v. a. die lokalen Verteilnetze haben. In diesem Kontext wird die Steuerung der Ladevorgänge als eine zentrale energiewirtschaftliche Herausforderung diskutiert. Aufgrund ihrer langen Standzeiten, großem Strombedarf und hoher Ladeleistungen, scheinen die Elektrofahrzeuge für eine Laststeuerung prädestiniert zu sein. Dazu können monetäre Anreizsysteme in Form variabler Stromtarife geeignet sein: Sie spiegeln die aktuelle Erzeugungssituation wider, informieren die Nachfrager und können daher ein entsprechendes Ladeverhalten auslösen.

Im vorliegenden Artikel wird das Wechselspiel zwischen variablen Stromtarifen und der Ladelast untersucht. Zur Analyse ist das Optimiermodell DS-Opt+ entwickelt worden. Es bildet insgesamt 4.000 Haushalte in zwei Wohnvierteln einer Großstadt bzgl. ihrer Stromnachfrage, ihres Mobilitätsbedarfs und ihrer Ausstattung mit PhotovoltaikAnlagen ab. Vier verschiedene Ladetarifmodelle werden

A.-G. Paetz $(\varangle)$ · T. Kaschub · P. Jochem · W. Fichtner Institut für Industriebetriebslehre und Industrielle Produktion (IIP), Lehrstuhl für Energiewirtschaft, Karlsruher Institut für Technologie (KIT), Hertzstr. 16, 76187 Karlsruhe, Deutschland

e-mail: alexandra-gwyn.paetz@kit.edu

T. Kaschub

e-mail: thomas.kaschub@kit.edu

P. Jochem

e-mail: patrick.jochem@kit.edu

W. Fichtner

e-mail: wolf.fichtner@kit.edu hinsichtlich ihrer Auswirkungen auf die Ladevorgänge und damit den Gesamtlastgang der Wohnviertel betrachtet.

Die Ergebnisse verdeutlichen, dass erst ab einer hohen Marktpenetration von Elektrofahrzeugen der Strombedarf nennenswert steigt und eine Ladesteuerung erfordern. Die im Modell getesteten Tarifmodelle sind zur Beeinflussung der Ladelast geeignet, wobei die lastabhängigen Tarifmodelle zu einer deutlichen Glättung des Lastgangs im Wohnviertel führen. Vor dem Hintergrund eines ausgeglichenen Lastgangs ist eine ungesteuerte Ladestrategie in unserer Analyse dem gesteuerten Laden über ein zeitabhängiges Tarifmodell sogar vorzuziehen. Aus den Ergebnissen lassen sich zahlreiche Implikationen für Wirtschaft und Wissenschaft ableiten.

Schlüsselwörter Elektromobilität .

Lastverschiebepotenziale · Ladestrategie · Variable

Tarifmodelle $\cdot$ Modellgestützte Optimierung

\section{Managing Charging Loads of Electric Vehicles by Monetary Incentives-A Model-Based Optimization}

Abstract Electric mobility is supposed to contribute to climate policy targets by reducing $\mathrm{CO}_{2}$-emissions in the transportation sector. Increasing penetration rates of electric vehicles $(\mathrm{EV})$ can lead to new challenges in the electricity sector, especially with regard to local distribution networks. Thus the management of charging loads is discussed as a key issue in energy economics. Due to their long parking times, high electricity and power demand, EV seem to be predestined for load management. Monetary incentives as dynamic pricing can be suitable for that: They reflect the current supply situation, pass the information to the consumers and can thus lead to a corresponding charging behaviour. 
In this article we analyse this interaction between dynamic pricing and charging loads. For this reason we have developed the optimization model DS-Opt + . It models a total number of 4,000 households in two residential areas of a major city with regard to its electricity demand, its mobility behaviour and its equipment of photovoltaic systems. Four different pricing models are tested for their effects on charging behaviour and thus the total load of the residential area.

The results illustrate that only fairly high penetration rates of EV lead to remarkably higher electricity demand and require some load management. The tested dynamic pricing models are suitable for influencing charging loads; load-based tariffs are best in achieving a balanced load curve. In our analysis uncontrolled charging strategies are superior regarding a balanced load curve than controlled strategies by time-varying tariffs. Our results lead to several implications relevant for the energy industry and further research.

Keywords Electric mobility · Load shifting potentials · Charging strategies $\cdot$ Dynamic pricing $\cdot$ Model-based optimization

\begin{tabular}{ll}
\multicolumn{2}{l}{ Abbreviations } \\
$K^{\mathrm{Ges}}$ & Strombezugskosten Gesamt \\
$E^{\mathrm{HH}}$ & Nachfrage Haushaltsstrom \\
$E^{\mathrm{E}-\mathrm{Fzg}}$ & Nachfrage Ladestrom \\
$V^{\mathrm{PV}}$ & PV-Vergütung \\
$p$ & Strompreis \\
$p^{\text {Auf }}$ & Strompreisaufschlag \\
$t$ & Zeitscheibe \\
$\mathrm{HH}$ & Haushalt
\end{tabular}

\section{Einleitung}

Als Mobilitätsform der Zukunft steht die Elektromobilität im Zentrum der aktuellen Diskussionen in Politik, Wirtschaft und Wissenschaft. Endliche Ölreserven, steigende Kraftstoffpreise und der fortschreitende Klimawandel infolge des verstärkten Ausstoßes von Treibhausgasen führen zu neuen umwelt- und wirtschaftspolitischen Rahmenbedingungen auf europäischer wie auf nationaler Ebene. Neben dem Ausbau Erneuerbarer Energien im Energiesektor soll im Transportsektor eine zunehmende Elektrifizierung den $\mathrm{CO}_{2}$-Ausstoß senken. Hierfür verfolgt die Bundesregierung das ambitionierte Ziel, bis ins Jahr 2020 eine Verbreitung von einer Million und bis ins Jahr 2030 von sechs Millionen elektrisch angetriebener Pkw auf Deutschlands Straßen zu erreichen (Bundesregierung 2009) ${ }^{1}$ - derzeit liegt sie noch

\footnotetext{
${ }^{1}$ Die Nationale Plattform Elektromobilität geht in ihrem letzten Bericht von einer Stückzahl von 600.000 Elektrofahrzeugen aus (NPE 2012).
}

bei 4.500 batterieelektrischen Pkw (0,01\%; KBA 2012a). Zwar gewinnen elektrisch angetriebene Zweiräder (insb. Pedelecs) derzeit an Beliebtheit, dennoch liegt der Anteil der Elektrokrafträder bei noch geringen 0,05 \% (KBA 2012b).

Eine verstärkte Verbreitung von Elektrofahrzeugen (E-Fzg) kann wesentliche Rückwirkungen auf das Energiesystem haben und die dortigen Herausforderungen, die durch eine zunehmende Elektrizitätsbereitstellung aus fluktuierenden Energiequellen und einer Einspeisung aus dezentralen Anlagen induziert sind, verschärfen. Zwar sind die Auswirkungen auf den zusätzlichen Strombedarf sowie ihre Bereitstellung über Kraftwerkskapazitäten eher gering (vgl. Pehnt et al. 2011), mit einer Erhöhung der abendlichen Lastspitzen ist vor allem auf Verteilnetzebene $\mathrm{zu}$ rechnen und gerade hier liegen die Herausforderungen (vgl. Blank et al. 2008; Jochem et al. 2012, Leitinger und Litzlbauer 2011; Shao et al. 2009). Da die Penetration von Elektrofahrzeugen in der Fläche nicht gleich verteilt, sondern sich zunächst aufgrund von bspw. Nachbarschaftseffekten wohl lokal konzentrieren wird (vgl. Cui et al. 2010), sind Netzüberlastungen insbesondere auf der Niederspannungsebene zu erwarten (Pollok et al. 2011).

Um dies zu vermeiden, sind Gegenmaßnahmen bei der Nachfrage - also bei den Haushalten - anzusetzen. Es werden dazu u. a. Steuerungsmechanismen für die Ladevorgänge der Elektrofahrzeuge in der Literatur diskutiert ohne, dass diese bereits explizit untersucht wurden (vgl. Blank et al. 2008; Brauner 2009; Leitinger et al. 2011; Nischler et al. 2011). Dazu gehören monetäre Anreizmechanismen in Form von Strompreissignalen (vgl. Link 2011). Variierende Strompreise sollen die aktuelle Erzeugungsund Netzsituation widerspiegeln, die Nachfrager darüber informieren und ein entsprechendes Ladeverhalten auslösen. Ziel ist eine geringere Nachfrage nach Ladeleistung bei hohem Strompreis und umgekehrt. Objektiv sind Elektrofahrzeuge für eine derart flexible Nachfrage prädestiniert, da sie einerseits den größten Teil des Tages geparkt sind (vgl. MOP 2009) und somit bei langen Standzeiten sich für eine Ladesteuerung anbieten und sie andererseits einen hohen Strombedarf ${ }^{2}$ haben. Damit sind die Kosteneinsparpotenziale durch das gesteuerte Laden höher als für andere Geräte im Haushalt. Ihre Anreizwirkung vorausgesetzt, haben variable Strompreise also das Potenzial Ladelasten zu steuern. Je nachdem wie diese Preise nun ausgestaltet werden, können mit einer flexiblen - in anderen Worten elastischen Nachfrage zwei Ziele verfolgt werden: zum einen kann ein ausgeglichener Lastgang erreicht werden, um in der Folge konventionelle Kraftwerke effizienter einzusetzen (wie das der Fall bei Niederpreistarifen für Nachtspeicherheizungen der Fall ist). Zum anderen kann eine bessere Integration des

\footnotetext{
${ }^{2}$ Bei einer durchschnittlichen Fahrleistung von $15.000 \mathrm{~km} / \mathrm{a}$ verdoppelt sich mit etwa $3.250 \mathrm{kWh} / \mathrm{a}$ die Haushaltsnachfrage.
} 
Tab. 1 Strukturierung beispielhafter Tarifmodelle (Paetz et al. 2012b)

\begin{tabular}{|c|c|c|c|c|c|}
\hline \multirow[t]{2}{*}{ Parameter } & \multicolumn{5}{|l|}{ Beispiele } \\
\hline & $\begin{array}{l}\text { Zeitabhängiger } \\
\text { Tarif } \\
\text { (HT/NT) }\end{array}$ & $\begin{array}{l}\text { Zeitabhängiger } \\
\text { Tarif } \\
\text { (Dynamisch) }\end{array}$ & $\begin{array}{l}\text { 3: Zeitabhän- } \\
\text { giger Tarif } \\
\text { (Echtzeit) }\end{array}$ & $\begin{array}{l}\text { 4: Lastabhän- } \\
\text { giger Tarif } \\
\text { (Hauslast) }\end{array}$ & $\begin{array}{l}\text { 5: Ereignis- } \\
\text { basierter Tarif } \\
\text { (CPP) }\end{array}$ \\
\hline Anzahl Preisstufen & Zwei & Drei & Unbegrenzt & Zwei & Zwei \\
\hline Verlauf der Preisstufen & $\begin{array}{l}\text { Täglich } \\
\text { gleich }\end{array}$ & $\begin{array}{l}\text { Täglich } \\
\text { verändernd }\end{array}$ & $\begin{array}{l}\text { Täglich } \\
\text { verändernd }\end{array}$ & Täglich gleich & $\begin{array}{l}\text { Täglich } \\
\text { gleich }\end{array}$ \\
\hline $\begin{array}{l}\text { Geltungsdauer pro } \\
\text { Preisstufe }\end{array}$ & $12 \mathrm{~h} / \mathrm{Tag}$ & Mind. $1 \mathrm{~h}$ & Variabel & l & $\begin{array}{l}\text { Ereignis- } \\
\text { basiert }\end{array}$ \\
\hline $\begin{array}{l}\text { Anzahl \& Höhe } \\
\text { von Lastgrenzen }\end{array}$ & I & l & l & $3 \mathrm{~kW}$ & l \\
\hline $\begin{array}{l}\text { Anzahl \& Dauer } \\
\text { kritischer Ereignisse }\end{array}$ & I & l & l & l & $\begin{array}{l}\text { Sechs pro } \\
\text { Jahr; jeweils } \\
2 \mathrm{~h}\end{array}$ \\
\hline Arbeitspreise (ct/kWh) & $15 ; 25$ & $15 ; 20 ; 25$ & $\begin{array}{l}\text { Frei zwischen } \\
10 \text { und } 40\end{array}$ & $\begin{array}{l}20 \text { (Basis); } \\
25 \text { (über der } \\
\text { Last-grenze) }\end{array}$ & $\begin{array}{l}20 \text { (Basis); } \\
40 \text { (während } \\
\text { kritischer } \\
\text { Ereignisse) }\end{array}$ \\
\hline
\end{tabular}

erneuerbaren Angebots erfolgen, wenn die Preise in dessen Abhängigkeit variieren (vgl. DoE 2006; Wellinghoff und Morenoff 2007). Damit können Elektrofahrzeuge im besten Fall sogar einen Beitrag zur Entlastung lokaler Stromnetze leisten.

In der vorliegenden Arbeit werden mehrere Tarifmodelle für Elektrizität konzipiert und ihre Auswirkungen auf die Ladelast analysiert. Dazu ist das gemischt ganzzahlige Optimiermodell DS-Opt,$+{ }^{3}$ das je 2.000 Haushalte in zwei Wohnvierteln bzgl. ihrer Stromnachfrage, ihres Mobilitätsbedarfs und ihrer Ausstattung mit Photovoltaik (PV)Anlagen abbildet, entwickelt worden. Die Verbreitung von E-Fzg entspricht ihrem technischen und wirtschaftlichen Potenzial, d. h. Haushalte verfügen dann über einen oder mehrere batterieelektrische Pkw (E-Pkw) und/oder Roller (E-Roller), wenn ihr Mobilitätsbedarf im Vergleich zum konventionell betriebenen Fahrzeug kostengünstiger gedeckt und die E-Fzg auf einem eigenen Stellplatz am Haus geladen werden können. Durch die Optimierung des Ladezeitpunktes der E-Pkw und E-Roller gemäß den Strompreisen, dem eigenerzeugten PV-Strom sowie dem Mobilitätsbedarf, werden die Auswirkungen der Tarifmodelle auf die Ladelast ersichtlich.

Bevor diese Ergebnisse in Abschn. 4 vorgestellt werden, werden in Abschn. 2 die Gestaltungsoptionen von Strompreismodellen erläutert und in Abschn. 3 das Optimiermodell vorgestellt. Der Beitrag endet in Abschn. 5 mit einer Ergebniszusammenfassung und -diskussion sowie einem Ausblick auf weiteren Forschungsbedarf.

\footnotetext{
${ }^{3}$ Demand Side Optimierung plus Elektromobilität und PV.
}

\section{Gestaltung von Tarifmodellen für Elektrizität}

Tarife für elektrische Energie haben eine Vielzahl an Anforderungen: verursachungsgerechte Weitergabe von Stromgestehungskosten und Netznutzung an die Abnehmer, effiziente Abrechnung der Stromabnahme bei einer hohen Anzahl an Kunden, verständlicher Aufbau sowie Beeinflussungspotenzial von Lastgängen (Klingenberg 1926). Die derzeit gängigen Tarifmodelle für Endkunden nutzen die Stromgestehungskosten (vom Kraftwerk bis zur Abrechnung) als Basis für die Kalkulation der Strompreise. Beim heute weitverbreiteten Grundpreistarif werden Vertriebs- und Leistungskosten über einen pauschalen Grundpreis und die Arbeitskosten über einen konstanten Arbeitspreis, der nach Kilowattstunden (linear, in der Regel mit degressivem Verlauf) abgerechnet wird, abgedeckt (vgl. Rehm 1999). Um die Chancen und Risiken aus den sich verändernden Bedingungen sowohl bei der Beschaffung als auch dem Transport bzw. der Verteilung an die Endkunden weiterzugeben, kann die Höhe des Arbeitspreises variiert werden. Dies kann grundsätzlich in Abhängigkeit des Nachfragezeitpunktes oder der Last geschehen. Zur Tarifgestaltung können dazu dann mehrere Parameter (bspw. welche Preisstufe gilt wann und für wie lange) variiert werden. Tabelle 1 stellt einige Beispieltarife im Überblick dar.

Die in der vorliegenden Studie betrachteten Tarifmodelle werden im Folgenden in Kürze dargestellt. Der pauschale Grundpreis wird nicht weiter berücksichtigt.

Tarifmodell 1: Konstanter Arbeitspreis

Der gegenwärtig verbreitete Grundpreistarif wird im Tarifmodell 1 abgebildet. Da der Arbeitspreis über einen län- 
Abb. 1 Abbildung des Spotpreises EEX und der gewählten Arbeitspreise im Tagesverlauf
Abb. 2 Lastvariabler Tarif mit zwei Preisstufen am Beispiel eines Haushaltes mit E-Fzg
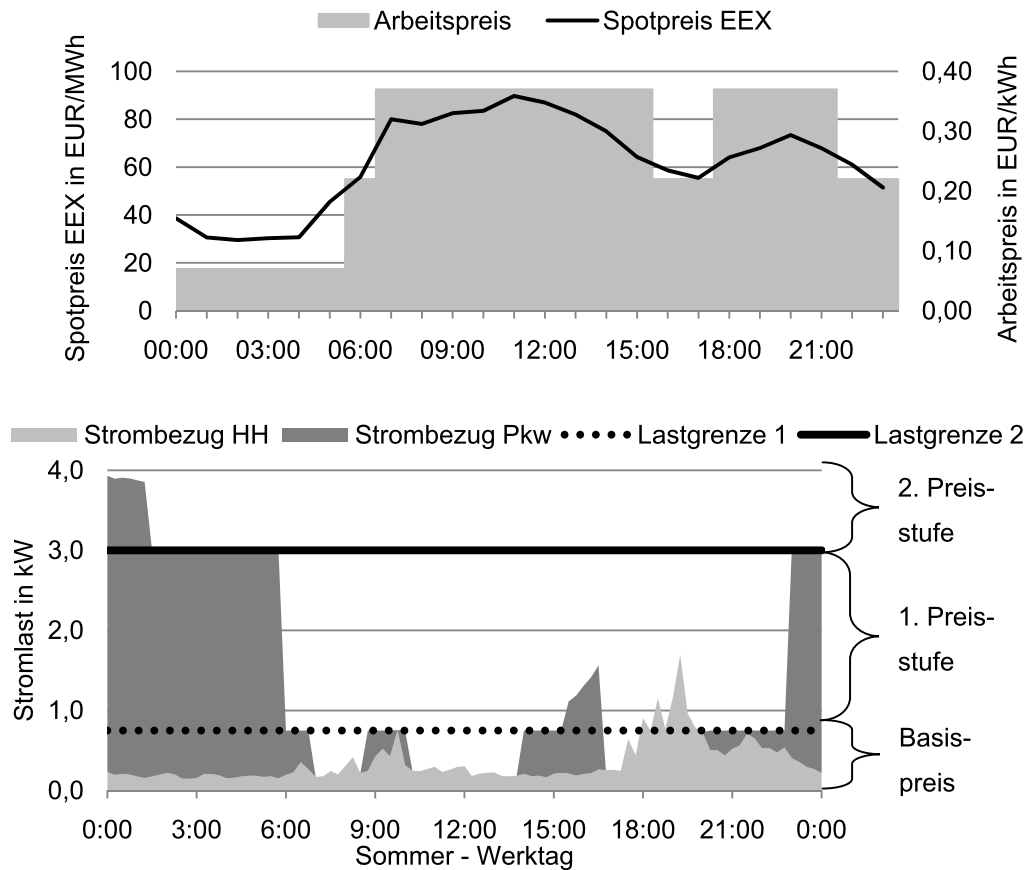

geren Zeitraum (in der Regel ein Jahr) konstant bleibt, besitzt dieser Tarif keine monetäre Anreizwirkung, den Ladevorgang zeitlich zu verschieben. Für diese Studie wird ein durchschnittlicher Arbeitspreis für Haushaltskunden von $22 \mathrm{ct} / \mathrm{kWh}$ herangezogen, der in etwa den aktuellen Arbeitspreis widerspiegelt (BDEW 2011).

\section{Tarifmodell 2: Zeitabhängiger Arbeitspreis}

Bei diesem Tarifmodell variiert der abgerechnete Arbeitspreis nach dem Zeitpunkt der Stromnachfrage. Die Höhe und zeitliche Varianz des Arbeitspreises spiegelt aktuelle Marktentwicklungen umso besser wider, je kürzer sie im Voraus kalkuliert werden. Im vorliegenden Fall werden Arbeitspreise in drei Preisstufen abgebildet, wobei die mittlere Stufe den Einheitspreis aus Tarifmodell 1 widerspiegelt. Die beiden anderen Stufen haben davon einen Abstand von je $15 \mathrm{ct} / \mathrm{kWh}$, um damit eine hohe Preisspreizung abzubilden. Diese Spreizung ist höher als die in derzeitigen Feldtests (vgl. Hillemacher et al. 2011) verwendeten. Der Verlauf dieser Arbeitspreise wird für jeden Tag in Abhängigkeit der gehandelten Preise an der Strombörse EEX aus dem Jahr 2008 kalkuliert. Dazu werden die Sportmarktpreise in drei Blöcke eingeteilt, die wiederum mit den drei Preisstufen des zeitabhängigen Tarifs versehen werden. Abbildung 1 zeigt beispielhaft einen daraus resultierenden Verlauf des zeitvariablen Arbeitspreises dargestellt an einem Apriltag.

Tarifmodell 3: Lastabhängiger Arbeitspreis

Im Fall eines lastabhängigen Arbeitspreises bildet die aktuelle Höhe der Haushaltslast die Grundlage für die Preishöhe. So wird in diesem Fall ein Preisaufschlag (um je $5 \mathrm{ct} / \mathrm{kWh}$ ) auf den konstanten Arbeitspreis (22 ct/kWh) berechnet, wenn die Haushaltslast (viertelstündliche Auflösung) definierte Lastgrenzen überschreitet. Eine erste Lastgrenze wird bei $0,75 \mathrm{~kW}$, eine zweite Lastgrenze bei $3 \mathrm{~kW}$ definiert. Diese Werte orientieren sich an der üblichen durchschnittlichen (Grenze 1) und Spitzenhaushaltslast (Grenze 2). Die sich daraus ergebenden Arbeitspreise zeigt Abb. 2 für einen beispielhaften Werktag im Sommer.

Tarifmodell 4: Zeit- und lastabhängiger Arbeitspreis Als vierte Option wird der Arbeitspreis sowohl nach dem Zeitpunkt als auch nach der Höhe der gegenwärtigen Haushaltslast variiert. Die Preise entsprechenden den Tarifmodellen 2 und 3. Der Arbeitspreis hat damit die drei Basispreisstufen in Höhe von 7, 22 und $37 \mathrm{ct} / \mathrm{kWh}$. Diese können jeweils um 5 oder $10 \mathrm{ct} / \mathrm{kWh}$ angehoben werden, sollten die entsprechenden Lastgrenzen überschritten werden.

\section{Vorstellung des Modells DS-Opt+}

Das Ziel des Modells DS-Opt+ ist die Minimierung der Strombezugskosten von jeweils 2.000 Haushalten eines Wohnviertels unter besonderer Berücksichtigung der Elektromobilität und der dezentralen Elektrizitätserzeugung mittels PV-Anlagen. In diesem gemischt ganzzahligen Optimierungsproblem sind für jeden Haushalt, der ein Elektrofahrzeug besitzt, die individuell preisgünstigsten Ladezeiten aus Endkundensicht zu finden. Im Modell wird der Zeitverlauf eines gesamten Jahres mittels neun Typwochen (jeweils drei Wochen zur Sommer-, Winter- und Übergangszeit) berücksichtigt, da insbesondere Strompreise, PV-Eigenerzeugung 
Abb. 3 Konzeptionelle Modelldarstellung

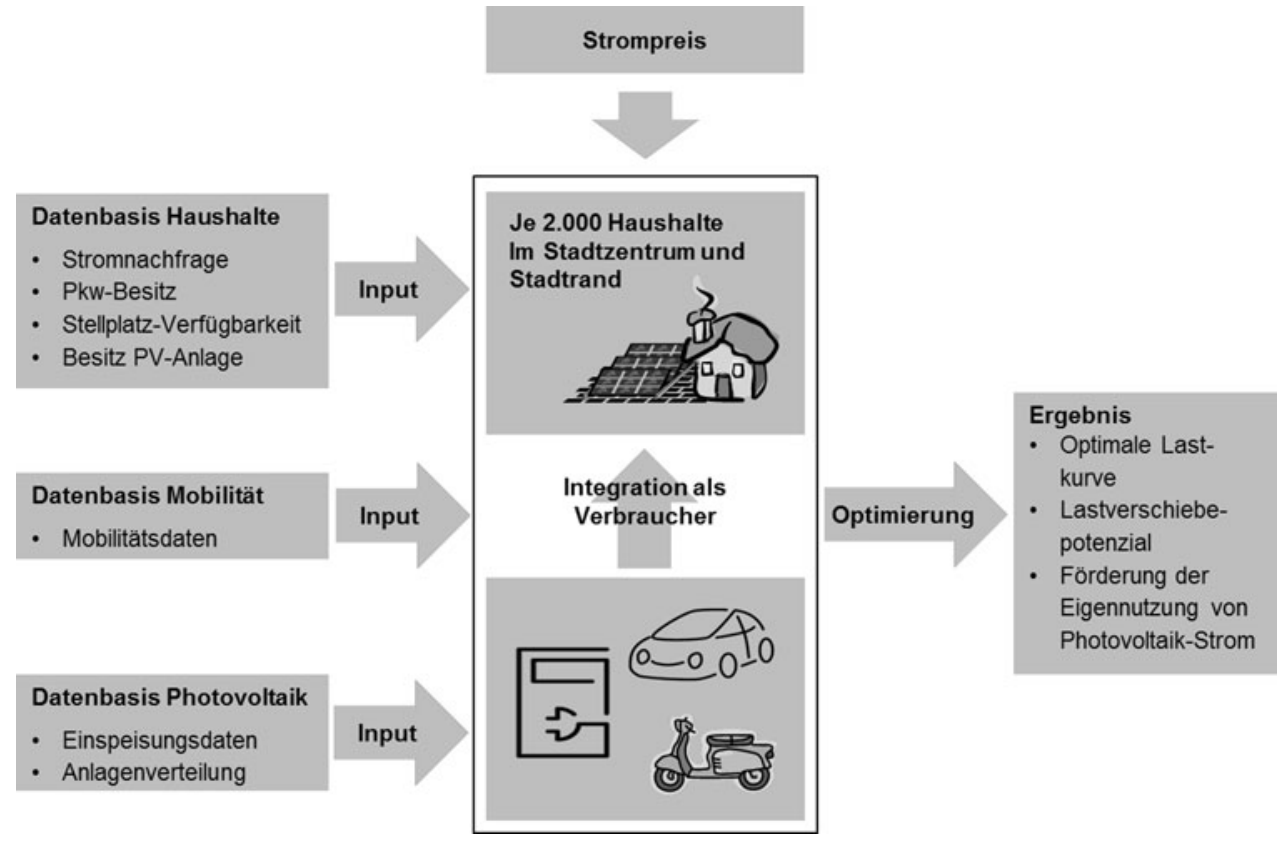

und der Haushaltsstrombedarf jahreszeitlichen Schwankungen unterliegen. Um die Ladevorgänge möglichst detailliert abzubilden, wird eine viertelstündliche Zeitauflösung gewählt. Eine direkte Hochrechnung auf Jahressummen ist dennoch nicht möglich, da die ausgewählten Typwochen keine Repräsentativität bezüglich der Jahreswerte erbringen können. Es werden daher pro Jahreszeit sowohl zwei extreme Wochen als auch eine durchschnittliche Woche dargestellt. Der Mobilitätsbedarf ist mit einer Typwoche integriert, da hier zunächst keine jahreszeitlichen Abhängigkeiten unterstellt werden.

Für die Berechnungen sind zukünftige Entwicklungen (insbesondere Mobilitätsbedarf, Strompreise sowie die Erzeugungsleistung) mittels verschiedener Szenarien vorgegeben. Um möglichst konsistente Zusammenhänge abzubilden, werden die historischen Daten für die EEX-Preise und die PV-Einspeisung aus dem Jahr 2008 genutzt. Die Mobilität beruht hingegen auf einem Betrachtungszeitraum von dreizehn Jahren (1997-2009). Jeder Haushalt agiert rational (Homo Oeconomicus) und unabhängig von anderen im Wohnviertel und wählt den Ladezeitraum, der ihn wirtschaftlich am besten stellt. Damit kann die maximale - pro Haushalt ökonomisch sinnvolle - Lastverschiebung realisiert werden. Der konzeptionelle Aufbau von DS-Opt+ wird in Abb. 3 darstellt.

\subsection{Beschreibung der Modellgebiete}

Als Erstnutzer von Elektrofahrzeugen werden Haushalte in städtischen Gebieten erwartet (Hebes et al. 2011). Die beiden modellierten Wohnviertel befinden sich daher in einer ausgewählten Großstadt (über 100.000 Einwohner). Dabei wird zwischen der Lage im Stadtkern und am Stadtrand unterschieden, da sich in der Mobilitäts- und Wohnstruktur Unterschiede ergeben, die für den Besitz und die Nutzung von Elektrofahrzeugen (bspw. Verfügbarkeit eigener Stellplätze) sowie für die PV-Thematik (bspw. Eigentümerquote) relevant sind.

Als Datenbasis sind die Rohdaten des Deutschen Mobilitätspanels (MOP 2009) aus den Jahren 1997 bis 2009 aufbereitet und ausgewertet worden. Im Gegensatz zu anderen Mobilitätsstudien werden bei der MOP Fahrten nicht nur an einem Stichtag pro Fahrzeug, sondern während einer Stichwoche pro Haushalt aufgezeichnet, so dass den Unterschieden im Wochenverlauf Rechnung getragen werden kann (vgl. Kloas et al. 2001).

Anhand der Merkmale Haushaltsgröße, Einkommensverteilung und Fahrzeugausstattung sind Unterschiede zwischen den beiden Modellgebieten abgebildet: Haushalte am Stadtrand sind tendenziell größer, verfügen über ein höheres Haushaltsnettoeinkommen und weisen einen höheren Ausstattungsgrad mit Fahrzeugen auf als Haushalte im Stadtkern. Entsprechend fällt der Mobilitätsbedarf am Stadtrand mit 86 km/Tag, der zu $57 \%$ mit einem Pkw gedeckt wird, auch höher aus als im Stadtkern mit $70 \mathrm{~km} / \mathrm{Tag}$ (davon $46 \%$ mit dem Pkw). Roller spielen in beiden Stadtgebieten mit 0,6 bzw. 0,9 km/Tag nur eine untergeordnete Rolle.

\subsection{Durchdringung der Elektrofahrzeuge in den Modellgebieten}

Der Mobilitätsbedarf spielt für die Betrachtung der Wirtschaftlichkeit von Elektrofahrzeugen eine bedeutende Rolle: Aufgrund der geringen operativen Kosten, rechnen sich 


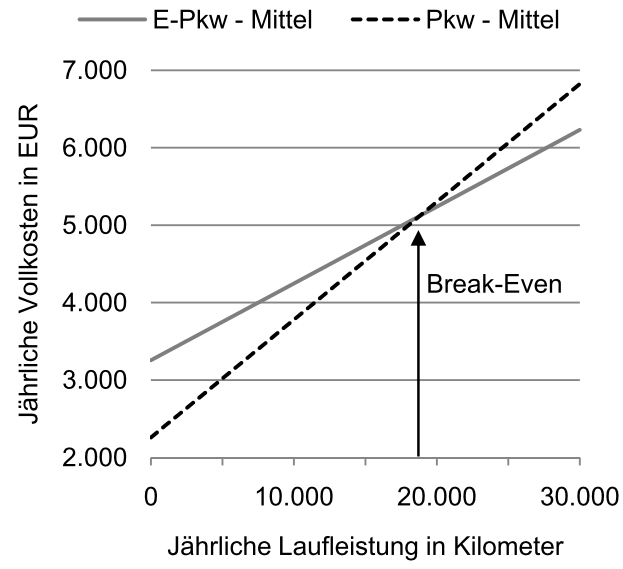

Abb. 4 Jährliche Vollkosten für Mittelklasse-Pkw in Abhängigkeit der jährlichen Kilometerlaufleistung (Erwartung 2020)

aktuell Elektrofahrzeuge für Haushalte mit hoher Kilometerleistung und einer Fahrdistanz im Rahmen der Batteriereichweite. Zwar spielen bei der Kaufentscheidung mehrere Kriterien eine Rolle (vgl. Caulfield et al. 2010), der Wirtschaftlichkeit wird aber für eine nachhaltige Verbreitung eine zentrale Rolle zugesprochen (vgl. Wietschel et al. 2012).

Daher wird im vorliegenden Fall in den Haushalten ein konventioneller Pkw (und/oder Roller) mit einem elektrisch Betriebenen ${ }^{4}$ substituiert, wenn einerseits der Mobilitätsbedarf elektrisch gedeckt werden kann und andererseits eine Wirtschaftlichkeit im Rahmen einer Haltedauer von zehn Jahren gegeben ist. ${ }^{5}$ Darüber hinaus ist ein eigener Stellplatz Voraussetzung, da die Elektrofahrzeuge nur zu Hause geladen werden, was den ersten Erfahrungen aus den verschiedenen Feldtests entspricht (vgl. Paetz et al. 2012a).

Die jährlichen Mobilitätskosten für konventionelle und für elektrisch betriebene Fahrzeuge werden in vier Segmenten (drei für Pkw, eine für Roller) im Rahmen einer $\mathrm{TCO}^{6}$-Analyse errechnet und miteinander verglichen. Der sich daraus ergebende Break-Even-Point stellt die für eine Substitution notwendige Kilometerlaufleistung dar. Wie Abb. 4 zeigt, ist für das Jahr 2020 (für das ausgereiftere Batterie- und Fahrzeugtechnik als heute erwartet werden) eine vollständige Amortisation im Mittelklasse-Segment ab einer jährlichen Laufleistung von etwa $18.600 \mathrm{~km}$ gegeben. Für die anderen Fahrzeugsegmente liegt der Break-EvenPoint für die jährliche Laufleistung ähnlich hoch.

In den modellierten Wohnvierteln ergibt sich ein Marktanteil von E-Pkw in Höhe von etwa zwei Prozent im Jahr 2010. Da davon auszugehen ist, dass sich im Zeitverlauf

\footnotetext{
${ }^{4}$ Es werden ausschließlich rein batteriebetriebene, keine hybriden (bspw. Plug-In Hybride) Fahrzeuge betrachtet.

${ }^{5}$ Die Präferenz für motorisierten Individualverkehr wird weiterhin unterstellt, so dass ein Ausweichen auf andere Verkehrsmittel (bspw. Radfahren) aus ökonomischen Gesichtspunkten nicht zugelassen wird. ${ }^{6}$ Total Cost of Ownership
}

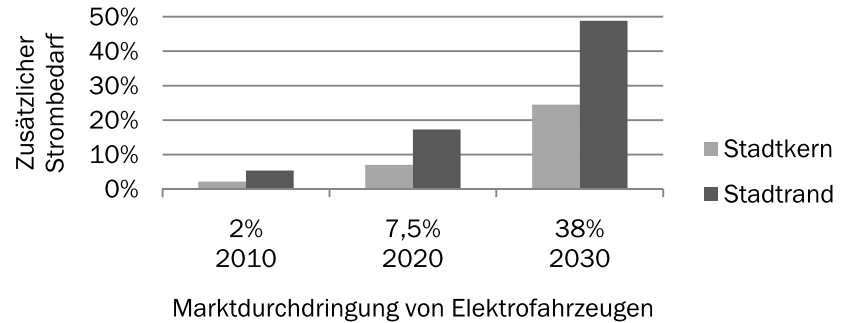

Abb. 5 Zusätzlicher Strombedarf (bezogen auf den Haushaltsstrombedarf in den Wohnvierteln) durch Elektrofahrzeuge in Abhängigkeit der Marktentwicklung

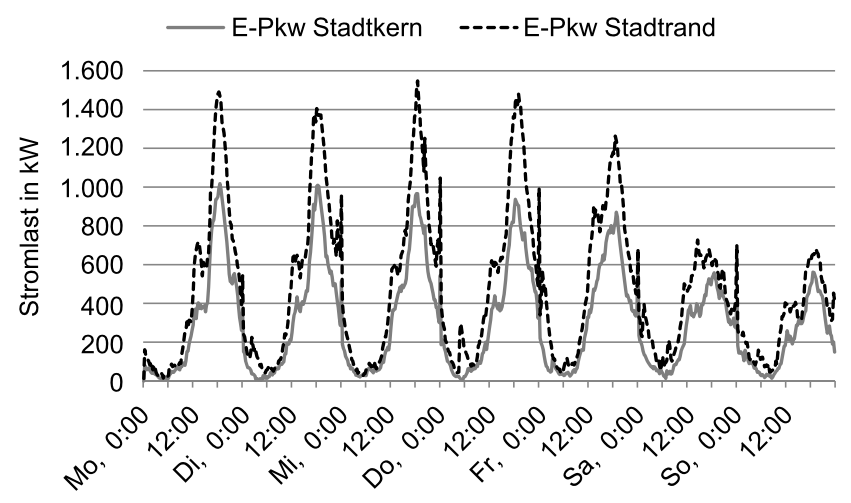

Abb. 6 Stromlast durch Elektrofahrzeuge in den Modellgebieten

Batterie- und Fahrzeugtechnik weiterentwickeln und dann mehr Pkw substituierbar sind, wird dies in einer Szenariobetrachtung mit drei Stützjahren für die nächsten 20 Jahre (2010, 2020, 2030) berücksichtigt. Der Marktanteil an E-Pkw steigt dann bis auf etwa $38 \%$ im Jahr 2030 an. ${ }^{7}$ Aufgrund der unterschiedlichen Charakteristika der Wohngebiete (bspw. höhere Stellplatzverfügbarkeit), werden dem Stadtrand mehr Elektrofahrzeuge zugeordnet. Daraus ergeben sich auch höhere Strombezugsmengen für die Ladevorgänge als im Stadtkern. Der zusätzliche Strombedarf durch Elektrofahrzeuge ist erst bei höheren Marktdurchdringungsraten (bspw. im Szenario 2030) relevant (vgl. Abb. 5). Bemerkenswert ist, dass fast $50 \%$ des Haushaltsstrombedarfs am Stadtrand zusätzlich an Ladeenergie anfällt.

Ein ähnliches Bild ergibt sich bei der Betrachtung der Ladelast im Wochenverlauf (vgl. Abb. 6). Die Elektrofahrzeuge werden unmittelbar nach dem Eintreffen zu Hause wieder aufgeladen. Die Lastspitze am Stadtrand übersteigt die Lastspitze im Stadtkern um rund $52 \%$. Es ist daher am Stadtrand mit stärkeren Auswirkungen auf das elektrische Energiesystem zu rechnen als im Stadtkern.

\footnotetext{
${ }^{7}$ Im Vergleich zu den Zielen der Bundesregierung ( $2 \%$ in 2020 und $12 \%$ 2030) wird die Marktdurchdringung im Modell aufgrund der Berücksichtigung des gesamten techno-ökonomischen Potenzials jeweils etwa zehn Jahre früher erreicht.
} 
Abb. 7 Stromlast Stadtrand (Winterwoche, Tarifmodell 1)

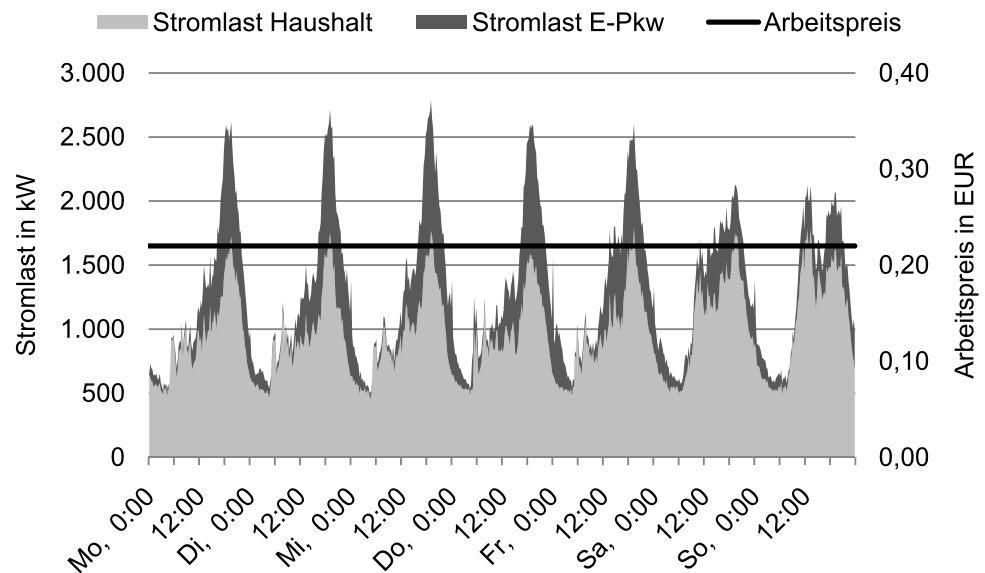

\subsection{Bestimmung der Strombezugskosten}

Die Strompreise spielen für die Entscheidung über den Ladezeitpunkt eine wichtige Rolle. Dazu werden die oben beschriebenen Tarifmodelle zu Grunde gelegt. Im Modell werden die Strombezugskosten aller Haushalte minimiert, was in den Zielfunktionen (vgl. Formel (1) und Formel (2)) abgebildet wird. Nicht dargestellt sind die Nebenbedingungen, in denen alle weiteren Zusammenhänge und Kriterien abgebildet sind. Dazu zählen das Mobilitätsverhalten und die technischen Daten der Elektrofahrzeuge, die Haushaltsanschlussleistung und die Vergütungssätze für die Eigennutzung sowie Netzeinspeisung von PV.

Formel (1): Tarifmodell 2 - Zeitabhängiger Arbeitspreis:

$K^{\mathrm{Ges}}=\sum_{\mathrm{HH}}\left[\sum_{t}\left[\left(E_{t, \mathrm{HH}}^{\mathrm{HH}}+E_{t, \mathrm{HH}}^{\mathrm{E}-\mathrm{Fzg}}\right) p_{t}-V_{t, \mathrm{HH}}^{\mathrm{PV}}\right]\right]$.

Formel (2): Tarifmodell 3 und 4 - Lastabhängiger und kombinierter Arbeitspreis:

$$
\begin{aligned}
K^{\mathrm{Ges}}= & \sum_{\mathrm{HH}}\left[\sum _ { t } \left[\left(\left(E_{t, \mathrm{HH}}^{\mathrm{HH}}+E_{t, \mathrm{HH}}^{\mathrm{E}-\mathrm{Hzg}}\right) p_{t}\right.\right.\right. \\
& \left.\forall E_{t, \mathrm{HH}}<0,1875 \mathrm{kWh}\right) \\
& +\left(\left(E_{t, \mathrm{HH}}^{\mathrm{HH}}+E_{t, \mathrm{HH}}^{\mathrm{E}-\mathrm{Fzg}}\right)\left(p_{t}+p^{\mathrm{Auf}}\right)\right. \\
& \left.\forall\left(E_{t, \mathrm{HH}} \geq 0,1875 \mathrm{kWh} \wedge E_{t, \mathrm{HH}} \leq 0,75 \mathrm{kWh}\right)\right) \\
& +\left(\left(E_{t, \mathrm{HH}}^{\mathrm{HH}}+E_{t, \mathrm{HH}}^{\mathrm{E}-\mathrm{Fzg}}\right)\left(p_{t}+2 p^{\mathrm{Auf}}\right)\right. \\
& \left.\left.\left.\forall E_{t, \mathrm{HH}}>0,75 \mathrm{kWh}\right)-V_{t, \mathrm{HH}}^{P V}\right]\right]
\end{aligned}
$$

\section{Ausgewählte Ergebnisse}

Im Folgenden werden ausgewählte Modellergebnisse vorgestellt. Da die resultierende Stromnachfrage (Haushaltsstrombedarf abzüglich PV-Eigennutzung) in den Winterwochen die der anderen Typwochen deutlich übersteigt, lassen sich die Modellergebnisse an den Winterwochen besonders anschaulich darstellen. Des Weiteren beschränken sich die hier vorgestellten Ergebnisse auf das Jahr 2030, da wie oben beschrieben, in diesem Szenario die größten Ladelasten auftreten. Aufgrund einer höheren Ausstattung mit Elektrofahrzeugen als auch mit PV-Anlagen wird zudem das Modellgebiet am Stadtrand in den Fokus genommen.

\section{Tarifmodell 1: Konstanter Arbeitspreis}

Bei einem konstanten Arbeitspreis besitzt der Haushalt keinen Anreiz den Ladezeitpunkt seines Elektrofahrzeuges anzupassen. Es wird daher ein ungesteuertes Ladeverhalten angenommen, bei dem das Fahrzeug unmittelbar nach jedem Eintreffen zu Hause an die Steckdose angeschlossen und aufgeladen wird. ${ }^{8}$ Lediglich Haushalte, die über eine PV-Anlage verfügen, haben einen monetären Anreiz ihr Ladeverhalten möglichst an die PV-Erzeugung zu koppeln, um von höheren Vergütungssätzen aufgrund der Eigenverbrauchsquote zu profitieren. ${ }^{9}$ In der Winterwoche ist dieser Einfluss allerdings zu vernachlässigen, da die Sonneneinstrahlung zur Abendzeit, wenn die meisten Elektrofahrzeuge zur Ladung bereitstünden, nachlässt. Es zeigt sich, dass sich die Ladeenergie mit der gewöhnlichen Haushaltsnachfrage überlagert und sich die Nachfragespitze in den Abendstunden um bis zu $57 \%$ erhöht (vgl. Abb. 7).

\section{Tarifmodell 2: Zeitabhängiger Arbeitspreis}

Beim zeitabhängigen Arbeitspreis wird versucht den Zeitpunkt der Ladevorgänge in die Stunden eines geringen Strompreises zu verlagern. Dadurch ergeben sich hier neue

\footnotetext{
${ }^{8}$ Die Haushaltsanschlussleistung $(3,7 \mathrm{~kW})$ entspricht der maximalen Ladeleistung. Es wird eine konstante Ladeleistung angenommen.

${ }^{9}$ Aufgrund des starken Zubaus von Anlagen zwischen 2009 und 2011 von ca. $18 \mathrm{GW}$ für die ein Eigennutzungsanreiz bestand, wird dies für alle PV Anlagen angenommen. Basierend auf EEG (2011) besteht ein monetärer Eigennutzungsanreiz i. H. v. 5,62 ct/kWh bzw. $10 \mathrm{ct} / \mathrm{kWh}$ bei einem Eigennutzungsanteil über $30 \%$.
} 
Abb. 8 Stromlast Stadtrand (Winterwoche, Tarifmodell 2)

Abb. 9 Stromlast Stadtrand (Winterwoche, Tarifmodell 3)

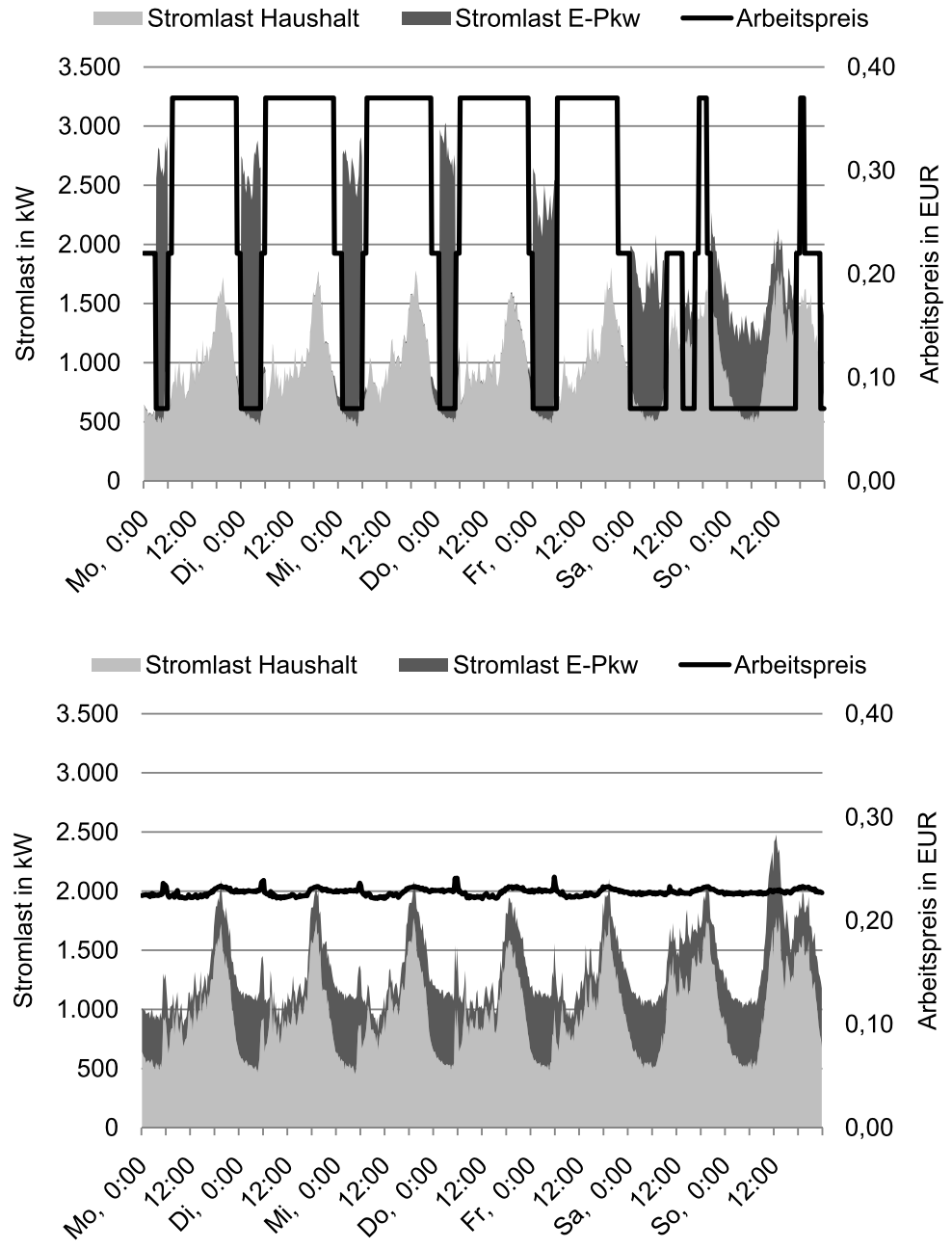

Nachfragespitzen in den Nachtstunden (vgl. Abb. 8). Diese übersteigen die Lastspitze der Haushaltslast um bis zu $68 \%$. Die maximale Stromlast steigt von $1.800 \mathrm{~kW}$ auf über $3.000 \mathrm{~kW}$ an.

\section{Tarifmodell 3: Lastabhängiger Arbeitspreis}

Die Einführung eines lastabhängigen Arbeitspreises vermeidet die bei den Tarifmodellen 1 und 2 auftretenden Nachfragespitzen, indem sie den Anreiz setzen, möglichst dann zu laden, wenn die Elektrizitätsnachfrage des Haushalts gering ist. Im Vergleich zur ungesteuerten Ladestrategie (Tarifmodell 1) kann die Last in der Spitze um $11 \%$ abgesenkt werden (peak shaving). Daraus ergibt sich eine Harmonisierung der Gesamtnachfragekurve. Da die Last in jedem Haushalt $\mathrm{zu}$ einem bestimmten Zeitpunkt variiert, schwankt der aggregierte Arbeitspreis zwischen 22, 27 bzw. $32 \mathrm{ct} / \mathrm{kWh}$ (vgl. Abb. 9).

\section{Tarifmodell 4: Zeit- und lastabhängiger Arbeitspreis} Variiert der Arbeitspreis in Abhängigkeit des Zeitpunktes und der Haushaltslast zeigen die Ergebnisse, dass die Nachfragekurve weiter harmonisiert wird, da die Ladeenergie nur noch zu Ausnahmezeitpunkten mit der Nach- frage im Haushalt zusammenfällt und es kaum zu Überlagerungen kommt (vgl. Abb. 10). Im Vergleich zur ungesteuerten Ladestrategie (Tarifmodell 1) kann die Last in der Spitze um fast $15 \%$ abgesenkt werden. Die noch auftretende Spitze am Sonntagvormittag ist neben dem günstigen Strompreis mit den längeren Freizeitfahrten an den Wochenenden zu begründen, die nur mit einer vollen Batterie zu schaffen sind. Dementsprechend ist es bei diesem kombinierten Tarif von Relevanz, die Preisspreizung und die Lastgrenzen aufeinander abzustimmen, um positive Effekte gegenüber den nicht kombinierten Tarifen zu erreichen.

\subsection{Vergleich der Strompreismodelle}

Ein Vergleich der Ergebnisse zeigt, dass durch Strompreissignale die Ladelast unterschiedlich beeinflussbar ist und Lastverschiebungen möglich sind. Dabei ist die Konzeption der Tarifmodelle entscheidend, da sie unterschiedliche Anreize setzen und daher auch verschiedene Auswirkungen haben. Nachfolgend in Tab. 2 sind die hier untersuchten Tarifmodelle gegenübergestellt. 
Abb. 10 Stromlast Stadtrand (Winterwoche, Tarifmodell 4)

Tab. 2 Vergleich der untersuchten Tarifmodelle ${ }^{\mathrm{a}}$

${ }^{a}$ Zur besseren Übersichtlichkeit der Ergebnisse werden die Werte indexiert dargestellt. Der Wert der maximalen Stromnachfrage ohne Elektrofahrzeuge stellt den Basiswert 100 dar

Tab. 3 Durchschnittliche Ladekosten (Winterwoche, Stadtrand)

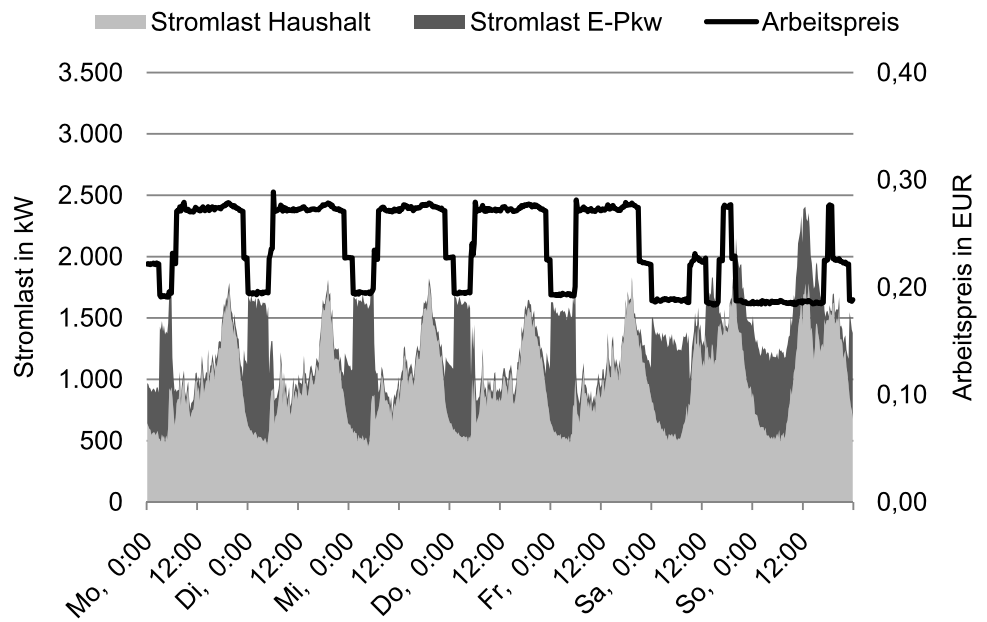

\begin{tabular}{llll}
\hline Szenario/Tarifmodell & $\begin{array}{l}\text { Maximale } \\
\text { Stromlast }\end{array}$ & $\begin{array}{l}\text { Minimale } \\
\text { Stromlast }\end{array}$ & $\begin{array}{l}\text { Grad der } \\
\text { Lastglättung } \\
\text { (Max/Min) }\end{array}$ \\
\hline Basis (Stromverbrauch ohne & 100,0 & 25,2 & 4,0 \\
Elektrofahrzeuge) & 154,8 & 27,0 & 5,7 \\
1: Konstanter Arbeitspreis & 168,3 & 28,7 & 5,9 \\
2: Zeitabhängiger Arbeitspreis & 116,9 & 36,1 & 3,2 \\
3: Lastabhängiger Arbeitspreis & 121,4 & 38,0 & 3,2 \\
4: Zeit- und lastabhängiger Arbeitspreis & & \\
\hline
\end{tabular}

\begin{tabular}{lll}
\hline Szenario/Tarifmodell & $\begin{array}{l}\text { Durchschnittliche } \\
\text { Ladekosten/E-Pkw } \\
{[\text { EUR] }}\end{array}$ & $\begin{array}{l}\text { Durchschnittliche } \\
\text { Ladekosten/kWh } \\
{[\text { EUR] }}\end{array}$ \\
\hline 1: Konstanter Arbeitspreis & 10,38 & 0,22 \\
2: Zeitabhängiger Arbeitspreis & 4,53 & 0,07 \\
3: Lastabhängiger Arbeitspreis & 11,41 & 0,23 \\
4: Zeit- und lastabhängiger Arbeitspreis & 10,04 & 0,20 \\
\hline
\end{tabular}

Die Integration von Elektrofahrzeugen führt in jedem Fall zu einer Erhöhung der Stromnachfrage und kann die Lastspitze sogar um bis zu $70 \%$ erhöhen. Da die Stromnachfrage im Haushalt von der Ankunft der Bewohner zuhause abhängt, ist diese mit der Nachfrage nach Ladestrom gekoppelt. Da sich dadurch im ungesteuerten Fall (Tarifmodell 1) nur die Nachfragespitzen (und nicht auch die Täler) erhöhen, verschlechtert sich der Harmonisierungsgrad der Nachfragekurve. Durch den zeitabhängigen Arbeitspreis (Tarifmodell 2), werden insb. die preisgünstigen Zeiten genutzt, um mit maximaler Leistung zu laden. Dadurch entsteht eine neue Lastspitze anstatt einer Lastglättung. Erst wenn der monetäre Anreiz (auch) von der Last abhängt, können die Nachfragespitzen abgeschwächt werden. Das geht im optimalen Fall mit der Auffüllung von Nachfragetälern einher, was insgesamt eine Harmonisierung der Gesamtnachfrage bewirkt. Der Harmonisierungsgrad wird im Vergleich zum Basisfall verbessert. Unterschiede zwischen Tarifmodell 3 und 4 sind in diesem Fall kaum vorhanden. Elektrofahrzeuge können mittels entsprechenden Tarifmodellen also auch als Mittel zur Nachfrageharmonisierung genutzt werden.

Beim Vergleich der Tarifmodelle hinsichtlich der resultierenden Ladekosten, entsteht ein etwas anderes Bild (vgl. Tab. 3). Nur wenn der Tarif (auch) zeitabhängig variiert, sind Kosteneinsparungen für die Haushalte möglich. Da der Arbeitspreis zum günstigsten Zeitpunkt beim Tarifmodell 2 lediglich $7 \mathrm{ct} / \mathrm{kWh}$ beträgt und damit die Arbeitspreise der anderen Tarifmodelle unterbietet, fallen die Ladekosten mit einer Senkung von durchschnittlich fast $60 \%$ am geringsten aus. Beim reinen lastabhängigen Tarif können Haushalte im 
Gegensatz zum konstanten Tarif ökonomisch nie besser gestellt werden, was zu einer Erhöhung der Ladekosten von durchschnittlich $10 \%$ führt.

\section{Diskussion}

\subsection{Zusammenfassung}

Um die Klimaschutzziele im Transportsektor zu erreichen, wird eine weitere Elektrifizierung des Transportsektors diskutiert. Die Verbreitung von Elektrofahrzeugen wird mit Auswirkungen auf das elektrische Energiesystem einhergehen. Bisherige Forschungsarbeiten in diesem Bereich kommen zum Ergebnis, dass die Steuerung der Ladevorgänge, bspw. über monetäre Anreizsysteme, notwendig ist, um eine systemverträgliche Integration der Elektrofahrzeuge zu gewährleisten. Der vorliegende Beitrag setzt an diesem Punkt an und analysiert, ob und in welchem Ausmaß monetäre Anreizsysteme in Form von variablen Strompreisen die Steuerung der Ladelast ermöglichen. Um dabei die maximal möglichen Lastverschiebungen zu untersuchen, wird unterstellt, dass sich Haushalte in Bezug auf das Ladeverhalten ihrer Elektrofahrzeuge rational verhalten.

Es wurde dazu ein Optimiermodell (DS-Opt+) entwickelt, das zwei Wohnviertel in einer Großstadt modelliert und in das Strompreise als Inputdaten eingehen. Dafür sind vier Tarifmodelle mit einem konstanten, einem zeitabhängigen, einem lastabhängigen und einem zeit- sowie lastabhängigen Arbeitspreis konzipiert worden.

Die Ergebnisse verdeutlichen, dass erst ab einer hohen Marktpenetration der Strombedarf nennenswert steigt und dies zunächst in Randlagen der Großstadt zu erwarten sind. Unter diesen Voraussetzungen wird eine Steuerung der Ladelast sinnvoll, da sich die Nachfrage nach Haushalts- und Ladestrom überlagern, was zu einer Erhöhung der Verbrauchspitzen führt. Die getesteten variablen Tarifmodelle sind zur Beeinflussung der Ladelast in unterschiedlicher Weise geeignet: Bei einem reinen zeitabhängigen Arbeitspreis können zwar die Haushalts- und Ladenachfrage entkoppelt werden, führen aber zu neuen und stärkeren Verbrauchsspitzen (zu Zeiten niedriger Arbeitspreise) als im ungesteuerten Fall. Gleichzeitig fallen die Ladekosten am geringsten aus, da die preisgünstigen Zeiten besser genutzt werden können.

Wird der Arbeitspreis in Abhängigkeit der anfallenden Haushaltslast variiert, so steigen die Ladekosten leicht an, da die Haushalte nie besser gestellt werden, als im Basisfall des konstanten Strompreises. Der lastabhängige Tariftyp führt aber zu einer gesamten Harmonisierung der Nachfragelast in den Wohnvierteln. Im Vergleich zum ungesteuerten Laden im Basisfall können die Lastspitzen um bis zu $15 \%$ gesenkt bzw. verlagert werden. Das entspricht in etwa den
Erfahrungen verschiedener Feldstudien im Haushaltssektor (12\%) und verdeutlicht das Lastverlagerungspotenzial eines Fahrzeuges gegenüber dem Gesamthaushalt mit all seinen Geräten (Stromback et al. 2011).

\subsection{Implikationen und Ausblick}

Aus den Ergebnissen lassen sich zahlreiche Implikationen für Wirtschaft und Wissenschaft ableiten. So wird deutlich, dass reine zeitabhängige Tarife bei einer hohen Konzentration von Elektrofahrzeugen eher kontraproduktiv für einen ausgeglichenen Lastgang sein können, da neue Lastspitzen auftreten können. Werden diese zeitvariablen Tarifmodelle auch für den Haushaltsstrom angeboten, können zudem die Lasten von Haushaltsgeräten (u. a. weiße Ware) mitverschoben werden, was die Auswirkung verstärkt. Die Spreizung zwischen Nachfragespitzen und -tälern würde sich sogar erhöhen. Dennoch ist der HT/NT-Tarif bereits vielen Kunden bekannt und mit relativ hohen Kosteneinsparungen verbunden. Gerade deswegen könnte auch eine hohe Verhaltenswirksamkeit erwartet werden.

Dynamischere Tarifmodelle, die die (Netz-)Last genauer in den Endkundenpreisen berücksichtigen, sind für eine Lastharmonisierung geeigneter. Die Einführung dieser geht aber mit zahlreichen Anforderungen einher. Um auf die variierenden Preise reagieren zu können, müssen aktuellen Preise und evtl. auch Lasthöhen jederzeit bekannt sein. Dies erfordert eine aktivere Kommunikation von Stromlieferanten zum Kunden sowie eine vollständige Transparenz und verständliche Visualisierung. Denkbar sind zahlreiche Informationskanäle, vom Online-Portal bis zu Anzeigengeräten im Haus und Auto. Für die Abrechnung solcher dynamischen Tarife, wird das digitale Messen der Elektrizitätsnachfrage unentbehrlich sein. Die Wirtschaftlichkeit sog. Smart Meter im Haus oder an Ladesäulen zu installieren und zu betreiben, muss - auch vor dem Hintergrund weiterer Vorteile für Energieversorgungsunternehmen und Kunden (bspw. regelmäßigere Abrechnungen) - überprüft werden. Hinzu kommen noch offene regulatorische Aspekte, wenn die Zähler direkt in den Fahrzeugen verbaut werden sollten (OnboardMetering).

Inwieweit diese dynamischeren Tarifformen von Endkunden verstanden und akzeptiert werden, ist hingegen offen. Zwar werden insbesondere zeitabhängige Tarifmodelle in vielen Feldstudien getestet, lastabhängige Tariftypen werden aber - außerhalb von Laborexperimenten - selten berücksichtigt (vgl. Paetz et al. 2012b). Insbesondere die Verhaltenswirksamkeit in Bezug auf die Ladevorgänge von Elektrofahrzeugen ist noch unerforscht. Im vorliegenden Modell ist aufgrund der Annahme wirtschaftlich rational handelnder Haushalte, von einer hohen Preiselastizität ausgegangen worden. Auch wenn diese Annahme in der Literatur für das Verhalten von Haushalten im Allgemeinen teilweise bestätigt wird (vgl. Bartusch et al. 2011), darf 
bezweifelt werden, ob es wirklich das reale Verhalten der Haushalte (auch langfristig) abbildet (vgl. Kim und Shcherbakova 2011). Dies zeigt eine Grenze dieser Arbeit auf, die sie mit allen Analysen, die sich auf Optimiermodellen stützen, teilt.

Für die weitere Forschung ist es daher wichtig, das reale Haushaltsverhalten langfristig auszuwerten und in solche Art von modellgestützten Analysen zu integrieren, um genauere Aussagen zu Lastverschiebepotenzialen - im Haushalt wie auch für Elektrofahrzeuge - treffen zu können. Die Ergebnisse bieten dann eine erweiterte Grundlage auch für Wirtschaftlichkeitsberechnungen zum Roll-Out IKTbasierter Infrastruktur. Neben den Smart Metern zählen hierzu auch Steuerungsmechanismen, bspw. intelligente Ladesäulen an denen die Fahrzeuge automatisiert systemschonend geladen werden. Mit solchen automatisierten Ladevorrichtungen sinkt die aktive Nutzerintegration und steigt die Wahrscheinlichkeit Verschiebepotenziale realisieren zu können. Dennoch wird zumindest die Einwilligung der Nutzer nötig sein, was die Akzeptanz solcher IKT-basierter Lösungen voraussetzt. Somit bedarf es an dieser Stelle ebenfalls weiterer Forschungsarbeiten.

Da mit einer vergleichsweisen höheren Verbreitung von Elektrofahrzeugen im gewerblichen als im privaten $\mathrm{Ge}$ brauch gerechnet werden kann, wird eine Integration des Sektors Gewerbe, Handel und Dienstleistungen (GHD) in das Modell DS-Opt+ in einem nächsten Schritt angestrebt. Neben E-Pkw und E-Rollern wird auch die Integration von Plug-In-Hybriden (PHEV) angestrebt, da aufgrund ihrer geringeren Reichweitenbeschränkung mit einer insgesamt höheren Marktpenetration von E-Fzg gerechnet werden kann. Die Annahmen bzgl. der Verbreitung von E-Fzg können aufbauend auf weiteren Forschungsergebnissen überprüft werden.

Zukünftig sollen auch Netzrestriktionen in die Tarifgestaltung einbezogen werden, um die lokale Netzsituation sowie die Abhängigkeiten zwischen den Haushalten zu berücksichtigen. Hinsichtlich des Harmonisierungsgrades erwarten wir eine Verbesserung sowohl für zeitabhängige als auch für lastabhängige Tariftypen. Damit blieben Stromtarife eine geeignete Variante, um Anreize zur Realisierung von Lastverschiebepotenzialen zu setzen.

\section{Literatur}

Bartusch C, Wallin F, Odlare M, Vassileva I, Wester L (2011) Introducing a demand-based electricity distribution tariff in the residential sector: demand response and customer perception. Energy Policy 39(2011):5008-5025. doi:10.1016/j.enpol.2011.06.013

BDEW (2011) Strompreisanalyse Haushaltsstrom zum 09.03.2011. Bundesverband der Energie- und Wasserwirtschaft. http:// bdew.de/internet.nsf/id/DE_20100311_PM_46_Prozent_des_ Strompreises_sind_Steuern_und_Abgaben/\$file/ Musterhaushalt $\% 202011 \% 20$ Unterteilung $\% 20$ staatliche $\% 20$ Steuern\%20und\%20Abgaben.pdf. Gesehen am 22. Mai 2012
Blank T, Mauch W, Mezger T, Wagner U (2008) Zusätzlicher Energie und Leistungsbedarf für Elektrostraßenfahrzeuge. Energiewirtsch Tagesfr 58(12):50-52

Brauner G (2009) Energiebereitstellung für die Elektromobilität. E\&I, Elektrotech Inftech 126(10):371-374. doi:10.1007/ s00502-009-0682-9

Bundesregierung (2009) Nationaler Entwicklungsplan Elektromobilität der Bundesregierung, Berlin

Caulfield B, Farrel S, McMahon B (2010) Examining individuals preferences for hybrid electric and alternatively fuelled vehicles. Transp Policy 17:381-387. doi:10.1016/j.tranpol.2010.04.005

Cui X, Lui C, Kim HK, Kao S-C, Tuttle M, Bhaduri BL (2010) A multi-agent-based framework for simulating household PHEV distribution and electric distribution network impact. TRB Comittee on Transportation Energy (ADC70)

DoE (2006) Benefits of demand response in electricity markets and recommendations for achieving them. A report of the US department of energy

EEG (2011) Gesetz für den Vorrang Erneuerbarer Energien (EEG). Novellierung 2011. http://www.gesetze-im-internet.de/ bundesrecht/eeg_2009/gesamt.pdf. Gesehen am 28.10.2011

Hillemacher L, Eßer-Frey A, Fichtner W (2011) Preis- und Effizienzsignale im MeRegio Smart Grid Feldtest - Simulationen und erste Ergebnisse. In: Proceedings of the 7th Internationale Energiewirtschaftstagung, Vienna, 2011

Hebes P, Trommer S, Kihm A (2011) Elektromobilität: Analyse des Marktpotentials in verschiedenen Raumtypen. DLR - Institut für Verkehrsforschung, Berlin

Jochem P, Kaschub T, Paetz A-G, Fichtner W (2012) Integrating electric vehicles into the German electricity grid—an interdisciplinary analysis. In: Proceedings of the 26th electric vehicle symposium, Los Angeles, USA

KBA (2012a) Bestand an Personenkraftwagen nach Kraftstoffarten zum 01.01.2012. Statistik des Kraftfahrt-Bundesamtes. http:// www.kba.de/cln_030/nn_269000/DE/Statistik/Fahrzeuge/ Bestand/EmissionenKraftstoffe/b_emi_z_teil_2.html. Gesehen am 22. Mai 2012

KBA (2012b): Bestand an Krafträdern nach Kraftstoffarten zum 01.01.2012. Anfrage beim KBA

Kim J-H, Shcherbakova A (2011) Common failures of demand response. Energy 36(2011):873-880. doi:10.1016/j.energy. 2010.12.027

Klingenberg G (1926) Bau großer Elektrizitätswerke. Springer, Berlin

Kloas J, Kuhfeld H, Kunert U (2001) Dynamik des Verkehrsverhaltens im Jahresvergleich. Analyse des deutschen Mobilitätspanels 1994 bis 1999. Gutachten des DIW im Auftrag des BMVBS

Leitinger C, Litzlbauer M (2011) Netzintegration und Ladestrategien der Elektromobilität. E\&I, Elektrotech Inftech 128(1-2):10-15. doi:10.1007/s00502-011-0800-3

Leitinger C, Litzlbauer M, Schuster A, Brauner G, Simic D, Hiller G et al. (2011) Smart Electric Mobility - Speichereinsatz für regenerative electrische Mobilität und Netzstabilität. Endbericht. Klimaund Energiefonds Österreich

Link J (2011) Elektromobilität und erneuerbare Energien: Lokal optimierter Einsatz von netzgekoppelten Fahrzeugen. Dissertation, TU Dortmund

MOP (2009) Das Deutsche Mobilitätspanel - Erhebungen der Jahre 1997 bis 2009. Institut für Verkehrswesen, Karlsruher Institut für Technologie, Karlsruhe

Nabe C, Beyer C, Brodersen N, Schäffler H, Adam D, Heinemann C et al (2009) Einführung von lastvariablen und zeitvariablen Tarifen. Studie im Auftrag der Bundesnetzagentur. http://www. bundesnetzagentur.de/cae/servlet/contentblob/153298/ publicationFile/6483/EcosysLasEcosysLastvariableZeitvaria 19042010pdf.pdf. Gesehen am 28. Oktober 2011 
NPE (2012) Fortschrittsbericht der Nationalen Plattform Elektromobilität (Dritter Bericht). Berlin 2012. http://www.bmu.de/files/pdfs/ allgemein/application/pdf/bericht_emob_3_bf.pdf. Gesehen am 18. September 2012

Nischler G, Gutschi C, Beermann M, Stigler H (2011) Auswirkungen von Elektromobilität auf das Energiesystem. E\&I, Elektrotech Inftech 128(1-2):53-57. doi:10.1007/s00502-011-0803-0

Newsham GR, Bower BG (2008) The effect of utility time-varying pricing and load control strategies on residential summer peak electricity use: a review. Energy Policy 38(2010):3289-3296. doi:10.1016/j.enpol.2010.01.027

Paetz A-G, Jochem P, Fichtner W (2012a) Demand Side Management mit Elektrofahrzeugen - Ausgestaltungsmöglichkeiten und Nutzerakzeptanz (Tagungsbandbeitrag, 12). In: Symposium Energieinnovation 2012, Graz

Paetz A-G, Kaschub T, Jochem P, Fichtner W (2012b) Demand response with smart homes and electric scooters-an experimental study on user acceptance. ACEEE Summer Study 2012, Pacific Grove

Pehnt M, Helms H, Lambrecht U, Dallinger D, Wietschel M et al (2011) Elektroautos in einer von erneuerbaren Energien geprägten Energiewirtschaft. Z Energiewirtsch 35:221-234

Pollok T, Matrose C, Dederichs T, Schnettler A, Szczechowicz E (2011) Classification and comparison of multi agent based control stratefies for electric vehicles in distribution networks. In: $21 \mathrm{st}$ international conference and exhibition on electricity distribution (CIRED 2011), Frankfurt, Germany

Rehm M (1999) Lastmanagement und dynamische Stromtarife in regenerativen Energieversorgungssystemen. Dissertation, Universität Karlsruhe

Sammer G, Meth D, Gruber CJ (2008) Elektromobilität - Die Sicht der Nutzer. E\&I, Elektrotech Inftech 125(11):393-400. doi:10.1007/s00502-008-0581-5

Shao S, Pipattanasomporn M, Rahman S (2009) Challenges of PHEV penetration to the residential distribution network. In: IEEE PES general meeting, S 1-8

Stromback J, Dromacque C, Yassin MH (2011) The potential of smart meter enabled programs to increase energy and system efficiency - a mass pilot comparison. VaasaETT global energy think tank

Wellinghoff J, Morenoff DL (2007) Recognizing the importance of demand response: the second half of the wholesale electric market equation. Energy Law J 28(2):389-419

Wietschel M, Dütschke E, Funke S, Peters A, Plötz P et al. (2012) Kaufpotenzial für Elektrofahrzeuge bei sogenannten ,early adoptern“. Endbericht einer Studie im Auftrag des BMWi. http:// isi.fraunhofer.de/isimedia/docs/e/de/publikationen/Schlussbericht_ Early_Adopter.pdf. Gesehen am 28. Mai 2012 


\section{Repository KITopen}

Dies ist ein Postprint/begutachtetes Manuskript.

Empfohlene Zitierung:

Paetz, A.-G.; Kaschub, T.; Kopp, M.; Jochem, P.; Fichtner, W.

Monetäre Anreize zur Steuerung der Ladelast von Elektrofahrzeugen : Eine modellgestützte Optimierung.

2013. Zeitschrift für Energiewirtschaft, 37.

doi:10.554/IR/1000077719

Zitierung der Originalveröffentlichung:

Paetz, A.-G.; Kaschub, T.; Kopp, M.; Jochem, P.; Fichtner, W.

Monetäre Anreize zur Steuerung der Ladelast von Elektrofahrzeugen : Eine modellgestützte Optimierung.

2013. Zeitschrift für Energiewirtschaft, 37 (1), 1-12.

doi:10.1007/s12398-012-0095-Z

Lizenzinformationen: KITopen-Lizenz 